\title{
XMM-Newton observation of the young open cluster Blanco $1^{\star}$
}

\section{X-ray time variability and flares}

\author{
I. Pillitteri ${ }^{1}$, G. Micela ${ }^{2}$, F. Reale ${ }^{1}$, and S. Sciortino ${ }^{2}$ \\ 1 Dip. di Astronomia, Università di Palermo, Piazza del Parlamento 1, 90134 Palermo, Italy \\ e-mail: pilli@astropa.unipa.it,reale@astropa.unipa.it \\ 2 INAF - Osservatorio Astronomico di Palermo, Piazza del Parlamento 1, 90134 Palermo, Italy \\ e-mail: giusi@astropa.unipa.it
}

Received 23 April 2004 / Accepted 14 September 2004

\begin{abstract}
We study the X-ray variability of the young open cluster Blanco 1 observed with the EPIC camera on board the $X M M$-Newton X-ray observatory. The time coverage of EPIC observations has allowed us to address short time scale (hours) variability, while the comparison with previous ROSAT observations has allowed us to investigate the variability on time scale of six years. On the time scale of hours, dM stars of the cluster are more variable than solar-mass stars. The main features of X-ray light curves in $\mathrm{dM}$ stars appear to be essentially flare-like events with a typical duration of the order of a few ks, while $\mathrm{dF}-\mathrm{dG}$ stars show smooth variations. Two intense flares were observed in the ZS76 cluster member. From the analysis of the light curves and of the time resolved X-ray spectroscopy we infer that they occurred in arcades, made of several similar coronal loops $\left(\sim 10^{10} \mathrm{~cm}\right.$ long), ignited at $\sim 15 \mathrm{ks}$ time delay. Minor heat pulses may occur in the first flare. The similarity of the two flares and of the flaring inferred structures may suggest that the flares are physically connected to each other. Amplitude variations on the time scale of 6 years are smaller by a factor 2 , whereas a factor of 10 is observed on the Sun in the same band. This implies a smaller amplitude of any analog of the 11-yr cycle in these young active coronae.
\end{abstract}

Key words. X-rays: stars - stars: activity - stars: flare - Galaxy: open clusters and associations: individual: Blanco 1

\section{Introduction}

This is our second paper based on XMM-Newton satellite observations of the young open cluster Blanco 1 . In the first paper (Pillitteri et al. 2004, hereafter Paper I) we presented the X-ray spectroscopy and photometry of the cluster. Here we address the issue of the coronal variability of the cluster stars within the same XMM-Newton observation, and on the longer time scale allowed by the comparison with ROSAT observations (Micela et al. 1999a). The variability in the X-ray band is linked to the dynamo mechanim which, in turn, is strictly related to the stellar structure and, probably, to the evolutionary status. In the Sun, it is possible to study in detail both the spatial structuring and the temporal changes on a range of time scales from a few minutes to several years. For all the other stars it is impossible to obtain this level of detail, but it is possible to infer the morphology and the physical parameters of the stellar coronae from the analysis of flares and of smooth modulation. Studies of classes of stars with the same age, metallicity and

^ Based on observations obtained with XMM-Newton, an ESA science mission with instruments and contributions directly funded by ESA Member States and NASA. environmental forming conditions, like open clusters, allow us to investigate if changes in the $\mathrm{X}$-ray variability are related to dynamo activity changes with stellar evolution.

The age of Blanco 1 is very similar to that of the well studied Pleiades cluster (around $100 \mathrm{Myr}$ ), while its metallicity is higher than that of Sun and of the Pleiades $([\mathrm{Fe} / \mathrm{H}]=+0.23$, Edvardsson et al. 1995; $[\mathrm{Fe} / \mathrm{H}]=0.14$, by following Jeffries et al. 1997). Its position - somewhat high above the galactic plane - is also noticeable and has suggested a peculiar origin, related to a high velocity progenitor cloud that ejected the cluster out of the plane after its formation (see Edvardsson et al. 1995). The high metallicity of this cluster and its age - very similar to that of Pleiades - allow to explore whether the X-ray activity is linked to its chemical composition or if the only leading parameter is the age through the relation between activity, rotation and dynamo efficiency.

Thanks to the large effective area of the EPIC instrument, the observations of the XMM-Newton satellite allow us to analyze for the first time in detail the X-ray light curves of the late type stars of Blanco 1 and to adress the short time scale variability. The comparison with previous ROSAT-HRI observations, taken in 1996 (Micela et al. 1999a), permits us to 
evaluate the variations in the X-ray luminosity on the time scale of $\sim 6 \mathrm{yr}$, which may be expected in analogy to the solar activity cycle.

The structure of the paper is the following: in Sect. 2 we summarize the processing of the data and of the source detection; in Sect. 3 we discuss the X-ray variability of Blanco 1 on both short time scales (hours) and long time scales (years); in Sect. 4 we present time resolved spectroscopy of two flares in a cluster late $\mathrm{G}$ star and we discuss the physics of the plasma involved and the morphology of the flaring region; in Sect. 5 we briefly discuss other sources variable on short time scales in the cluster region; Sect. 6 summarizes our results.

\section{X-ray observation and preliminary data analysis}

Here we summarize the XMM-Newton observation and the preliminary processing of the data. Further details are given in Paper I, Sect. 2. The observation was carried out during the revolution number 0461 of the XMM-Newton satellite in June 2002 for a nominal duration of $50 \mathrm{ks}$. The pointing, $\mathrm{RA}=0^{\mathrm{h}} 02^{\mathrm{m}} 48^{\mathrm{s}}$ and Dec $=-30^{\circ} 00^{\prime} 00^{\prime \prime}(\mathrm{J} 2000)$, is the same as one of the previous ROSAT observations of this cluster (Micela et al. 1999b). The ROSAT observation of this region was carried out with the HRI between Dec. 1995 and Nov. 1996. Three segments were acquired for a total of $88 \mathrm{ks}$. Due to the low detector effective area and to the distance of the cluster, the ROSAT-HRI did not allow a good timing analysis.

The source detection, described in Paper I, on the sum of MOS and $p n$ data led to 190 X-ray sources, 36 of them matching with known cluster members. The sensitivity varies throughout the field of view, the lowest value of $\log L_{X}$ for detected cluster sources being 28.35 at the distance of $250 \mathrm{pc}$ assumed for Blanco 1. In this work we focus our discussion on the results from $p n$ data analysis, because among the EPIC instruments it is the most sensitive one. We have conducted the source detection on the $p n$ image and obtained a list of 152 X-ray sources; 33 of them match cluster stars in the optical band. One cluster star is undetected with $p n$, while two other cluster stars lie only in the MOS field of view. To assess the short time scale variability, we have used the one sample Kolmogorov-Smirnov (KS) test on the unbinned photon arrival times. This analysis has been applied to all the sources detected in the $p n$ data, independently of the cluster membership status of their optical counterparts. We have selected the photons in a circle of radius $20^{\prime \prime}$, centered on the position of the detected sources. The radius is a good compromise between the need to collect enough counts for faint sources and the need to minimize the contamination by background and by nearby sources. To test the background variability we have applied the KS test on the photon arrival times comprised between $30^{\prime \prime}$ and $80^{\prime \prime}$, after exclusion of photons of the sources. We define as variable a source (and, with the same criterion, the local background) for which the KS test reports a probability of constant rate $\leq 1 \%$; the number of variable sources found with this criterion is 22 out of 152 sources detected with $p n$ instrument. Out of the 22 variable sources, 14 are cluster members. By carrying out a similar analysis on the MOS data, the two stars detected with only the MOS CCD have been found not to be variables.
For the long time scale variability, we have compared the X-ray luminosities $L_{\mathrm{X}}$ with those obtained in the previous ROSAT-HRI observation (Pillitteri et al. 2003). Because of the different energy bands of ROSAT (0.1-2.4 keV) and XMM-Newton (0.3-5.0 keV) observations, we have recalculated the XMM-Newton $L_{X}$ values starting from the EPIC MOS count rates reported in Paper I in $0.1-2.4 \mathrm{keV}$ by assuming a single temperature Raymond-Smith thin plasma emission model at $1 \mathrm{keV}, \mathrm{N}_{\mathrm{H}}=3 \times 10^{20} \mathrm{~cm}^{-2}$, as done by Pillitteri et al. (2003) to derive ROSAT $L_{X}$ values.

\section{Results: The X-ray variability of Blanco 1}

In this section we discuss the X-ray variability of Blanco 1 stars on both short time scales (hours), within our XMM-Newton observation, and on longer time scales ( $\sim 6$ years), by comparison of the EPIC data with the previous ROSAT-HRI ones (Micela et al. 1999a; Pillitteri et al. 2003).

\subsection{Short time scale variability}

Among Blanco 1 members we find 14 X-ray variable sources, the number of variable stars per spectral type are: $\mathrm{A}=1, \mathrm{dF}=$ $0, \mathrm{dG}=3, \mathrm{dK}=4$ and $\mathrm{dM}=6$, respectively; the number of detections in the pn data among these types are: $3,2,6,14$ and 8 respectively, thus yielding the fractions of variable stars: $33 \%, 0 \%, 50 \%, 29 \%$ and $75 \%$. Variability on short time scale appears more frequent in $\mathrm{dG}-\mathrm{dM}$ Blanco 1 stars. This may be biased by the easier detection of variability in high statistics sources, since $\mathrm{dG}-\mathrm{dK}$ types show higher X-ray activity with respect to A and early $\mathrm{dF}$ types. Moreover, it is worth noting that for $\mathrm{dM}$ stars, being detected only when they are flaring, the fraction exhibiting variability is even higher than that of the more X-ray bright $\mathrm{dG}$ and $\mathrm{dK}$ members of the cluster.

The light curves of the variable sources in the cluster are shown in Figs. 1 and 2, the times being counted from the start of the observation. To produce the curves, we have considered the arrival times of all events in a $20^{\prime \prime}$ circle centered on source positions, without background subtraction. The background rate in the $0.3-5.0 \mathrm{keV}$ and in the extraction circle ranges from 0.7 to $2.8 \mathrm{cts} / \mathrm{ks}$. The temporal bin size has been chosen depending on source count statistics (at least 5 counts per bin), and it is invariably $\gtrsim 700 \mathrm{~s}$. The light curve of the whole pn image is flat except for two intervals at $\sim 18$ and $\sim 32 \mathrm{ks}$ for a total of $\sim 0.8 \mathrm{ks}$. We have discarded these intervals before to accumulate the source light curves. Furthermore, the bins with large errors were omitted in the plots for sake of clarity. The small gap at $\sim 8 \mathrm{ks}$ is due to a break in the observation time.

The proximity to a chip gap and to another faint source could have influenced the evaluation of variability and the light curve of BLX 24. Stars P3, P5, BLX 37, BLX 46, BLX 17, ZS 76 and probably $Z S 45$ show flare-like impulsive events with duration of a few ks each. In particular, ZS76 exhibits two intense flares whose detailed analysis is presented in Sect. 4. Stars $B L X 37, B L X 46$ and $B L X 17$ show a flare followed by secondary peaks. For $B L X 37$ we can identify a main flare after $\sim 7 \mathrm{ks}$ and another bump superimposed on its decay phase at $16 \mathrm{ks}$. The flare occurring on $B L X 46$ after $\sim 15 \mathrm{ks}$ shows 

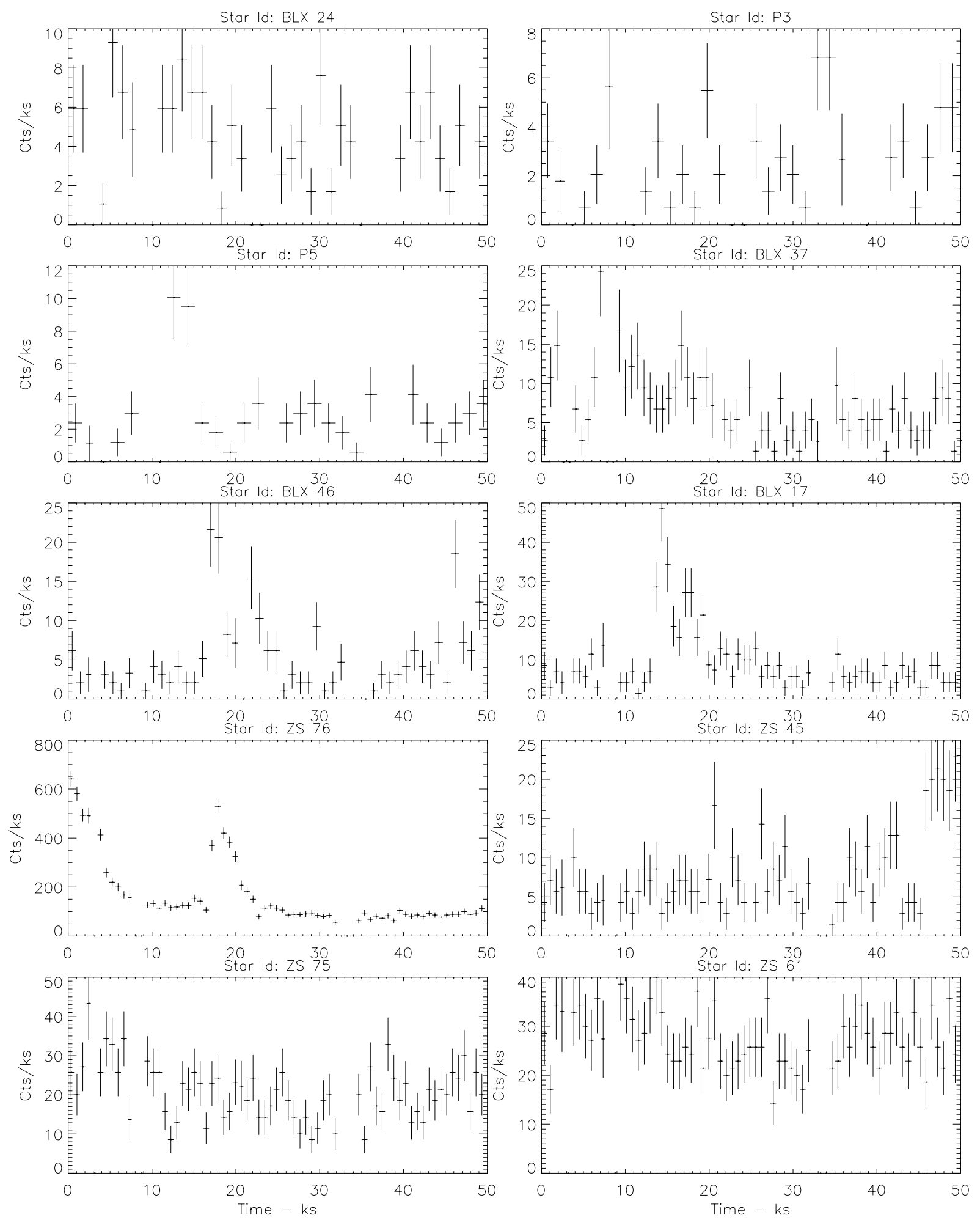

Fig. 1. Light curves of Blanco $1 \mathrm{X}$-ray variable stars.

a secondary bump at $\sim 22 \mathrm{ks}$, and it is similar to the one on $B L X$ 17. In the latter we clearly see a flare at $\sim 12 \mathrm{ks}$ followed by a second peak. The two peaks are separated by a dip of $\sim 1.5 \mathrm{ks}$. This kind of evolution is present also in the combined MOS light curve, although less pronounced for the lower count statistics. Hence we conclude that this temporal structure is real and not an instrumental artifact. The quiescent emission is comparable with the background rate so that the detection of BLX 17 is due essentially to its flare. The second peak that follows the main flare maximum could be interpreted as a secondary event in the same region of the corona triggered by the first event (Reale et al. 2004).

On a time scale of the order of the exposure time, a smooth, small-amplitude variation can be noticed in ZS 75 and ZS 61, 

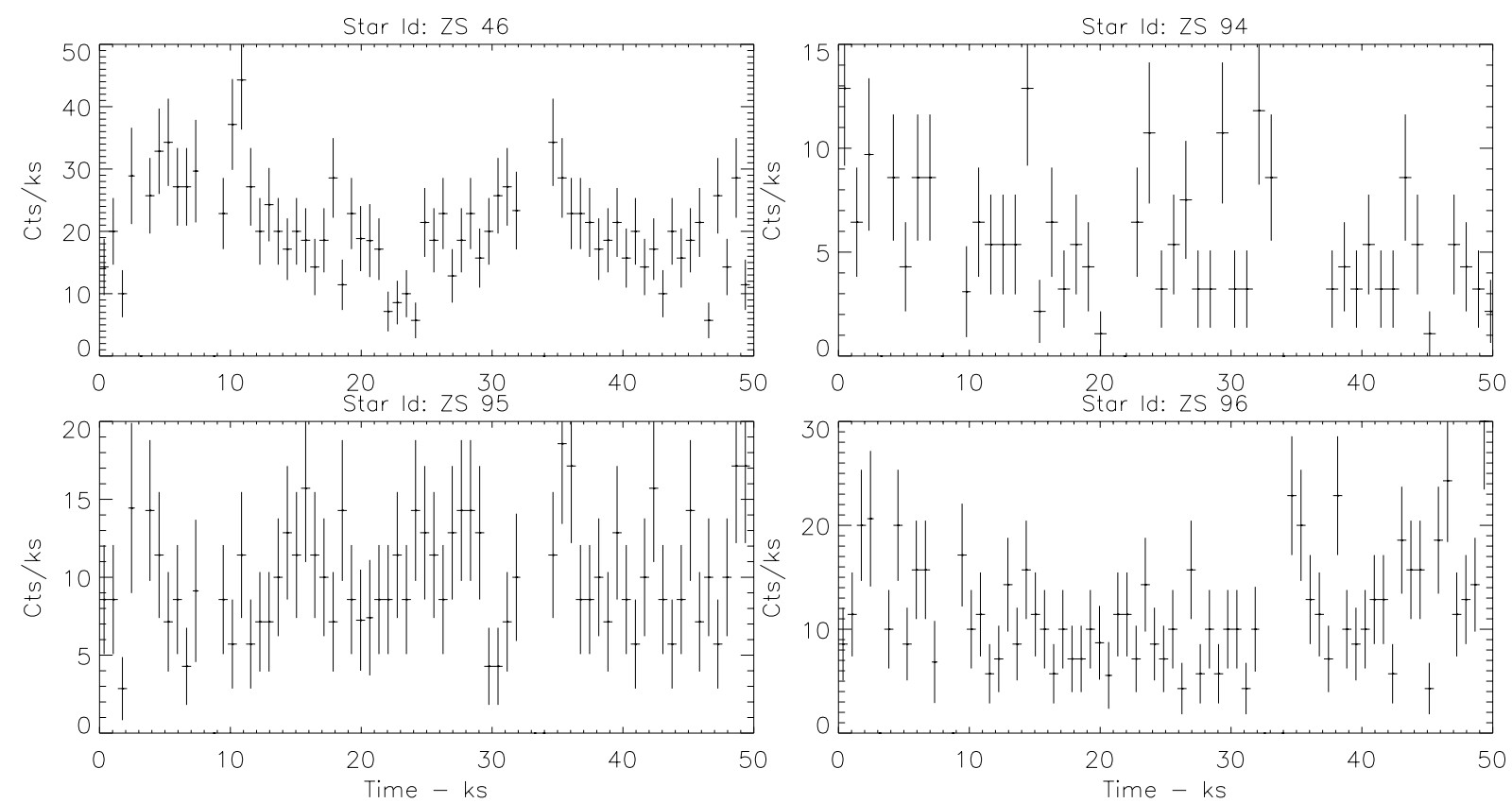

Fig. 2. Same as in Fig. 1.

with a higher emission at the extremes of the observation. In ZS 75 some impulsive events are also recognizable, as well as dips at 12 and $28 \mathrm{ks}$. The X-ray light curve of ZS 46 shows a smooth modulation with a characteristics time of $\sim 20 \mathrm{ks}$. This variation could be due to an inhomogeneous distribution of emitting structures. It is interesting to note that, as derived in Paper I, $\log L_{\mathrm{X}} / L_{\mathrm{bol}}$ of this star is equal to -3 , the saturation limit observed in active stars. The super-saturated star VXR45, in the very young open cluster IC 2391, is reported by Marino et al. (2003) to exhibit clear rotational modulation. This supersaturation does not appear to be linked to a uniform distribution of equally emitting structures, rather the authors make the hypothesis that the simultaneous presence of a X-ray bright active region at low latitudes and a less X-ray bright polar active region determine the observed periodic variations. As an explanation, Jardine (2004) suggests that rapid rotators like AB Dor and VXR45 experience a centrifugal stripping of coronal loops which break and open toward the outer space thus becoming X-ray dark regions.

The stars in which we detect smooth variations have masses in the $0.7-1 M_{\odot}$ range, while stars which show mainly or only flares are less massive, typically dM type stars. If a modulation were present in these low mass stars, due to their lower "quiescent" emission, it could be hardly detected, with flares being the only detectable features of variability. Light curves of ZS 94, ZS 95, ZS 96 can be less clearly classified, because their variability can be due either to flares or to smooth large scale variations.

In summary, the light curves discussed above show that rapid and subsequent flares are the typical features occurring in the low mass stars, while the solar-mass stars show smoother variations, with less frequent flares. The occurrence of subsequent flares in at least three $\mathrm{dM}$ stars suggest that in active young stars such flares could be triggered in nearby coronal regions by an initial event. We discuss this interpretation further in Sect. 4 along with the analysis of the flares that occurred in ZS 76.

\subsection{Long time scale variability}

The comparison between the EPIC-XMM and the HRI-ROSAT $\mathrm{X}$-ray luminosities of Blanco 1 stars allows us to evaluate their $\mathrm{X}$-ray variability on a time scale of about $6 \mathrm{yr}$ (i.e. one half of the solar cycle). The scatter plot in Fig. 3 shows the $L_{X}$ values of Blanco 1 stars observed with EPIC-XMM and HRI-ROSAT. The arrows indicate the sources detected only in the EPIC observation and the lines trace the equal, twice and half $L_{X}$ ratio loci. For ZS 76 we show both the quiescent and the average luminosity including the flares (marked by an asterisk). None of the stars undetected in the XMM-Newton observation has been detected in the previous ROSAT observation. It is highly unlikely that all the stars have changed their luminosities in a coherent way, hence we expect to observe a scatter around the equal ratio line. The absence of a systematic offset between XMM-Newton and ROSAT luminosities, in spite of the significant difference in the energy band pass confirms the substantial correctness of the model assumed in Micela et al. (1999a).

On the time scale of $\sim 6 \mathrm{yr}$ the variability amplitude in Blanco 1 is within a factor of two for all spectral types, ranging from A to dM. On a similar time scale and in a very similar soft X-ray band, the Sun shows larger amplitude variations, with a median value $\gtrsim 1$ dex due to the presence of the $11-y r$ cycle (Micela \& Marino 2003). The differences in stellar structure between $\mathrm{dF}-\mathrm{dG}$ and $\mathrm{dM}$ stars do not seem to imply a significant difference in the long term X-ray variability, despite models predicting different dynamo mechanisms. The internal structure of dG stars should produce an $\alpha-\omega$ dynamo at the base of the convective zone while, in the fully convective dM stars, 


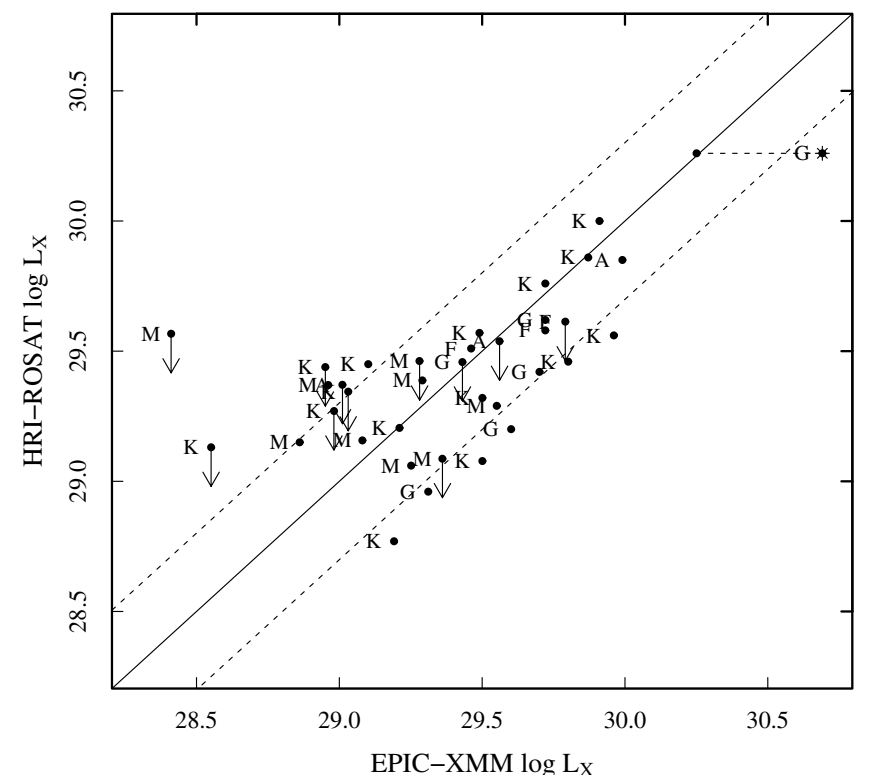

Fig. 3. Scatter plot of $L_{\mathrm{X}}$ obtained from the EPIC-XMM and the HRI ROSAT observations of Blanco 1. Capital letters indicate the spectral types, diagonal lines trace the equal, twice and half ratio loci. For the flaring star ZS76, discussed in Sect. 4, we marked the quiescent X-ray luminosity (open circle) and the luminosity averaged over the whole XMM-Newton observation including the flares (asterisk). Arrows indicate upper limits in ROSAT observation that are detections in the XMM-Newton observation.

a turbulent dynamo could be present, generating small scale coronal structures and should not lead to analogs of the solar cycle. Stern et al. (1995) made the hypothesis of a turbulent component in the dynamo of young $\mathrm{dG}$ type Hyades stars to explain the lack of strong, long term variability. Figure 3 suggests that this turbulent dynamo component - if present also in young solar-type stars - could have a primary role in young coronae like those of Blanco 1 stars, thus masking the evidence of cycles due to the $\alpha-\omega$ component. Other studies on nearly coeval open clusters, based on ROSAT and XMM-Newton data (e.g. Pleiades, Gagné et al. 1995; NGC 2516, Pillitteri et al. 2004), confirm that on these time scales active, young stars are much less variable than the Sun. Were stellar cycles present in young, active stars, they should have amplitude variations much smaller than in the Sun, according to the suggestion of Stern et al. (1995).

\section{The flares}

Flare events can give insight into the coronal structure in which they occur. Differently from the Sun, on stars it is impossible to resolve spatially the flaring region but, by making an analogy with the solar flares and use of flare loop modelling, it is possible to infer the typical sizes and the morphology of the loop structures involved in stellar flares.

Reale et al. (1997) presented a method to infer the geometrical size and other relevant physical parameters of the flaring loops, based on the decay time and on the evolution of the temperature and of the emission measure $(E M)$ during the flare decay. The method has been further extended for its application to
ROSAT observations by Reale \& Micela (1998) and it has been applied later to analyze several other stellar flares (e.g. Favata et al. 2001, BeppoSAX data; Briggs \& Pye 2003; and Stelzer et al. 2002, XMM-Newton data).

Reale et al. (1997) provide an empirical formula for the estimation of the unresolved flaring loop length, which is essentially derived from the thermodynamic e-folding decay time of a semicircular flaring loop with constant cross-section (Serio et al. 1991):

$$
L_{9}=\frac{\tau_{L C} \sqrt{T_{7}}}{120 F(\zeta)} \quad F(\zeta) \geq 1
$$

where $L_{9}$ is the loop semi-length in units of $10^{9} \mathrm{~cm}, T_{7}$ the loop maximum temperature at the flare peak in units of $10^{7} \mathrm{~K}$, $\tau_{\mathrm{LC}}$ the decay time derived from the light curve, and $F(\zeta)$ a non-dimensional correction factor larger than one (i.e. a shorter loop length) if significant heating is present during the decay. The slope $\zeta$ of the decay path in the density-temperature diagram (Sylwester et al. 1993) is maximum $(\sim 2)$ if the heating is negligible in the decay and minimum $(\sim 0.5)-$ the slope of the loci of the hydrostatic loops with decreasing temperature - if the heating dominates the decay (Sylwester et al. 1993). From Eq. (1), the slower the decay (or the higher the temperature) the longer is the loop. The effect of the heating is to delay the decay phase or, conversely, a given decay time may be compatible with a slowly decaying heating in a shorter loop.

The correction factor $F(\zeta)$ is calibrated on the observation instrument and for EPIC pn we have used (Reale et al. 2004):

$F(\zeta)=c_{a} \exp \left(-\zeta / \zeta_{a}\right)+q_{a}$

where

$c_{a}=11.6 \pm 0.5 \quad \zeta_{a}=0.56 \pm 0.06 \quad q_{a}=1.2 \pm 0.1$

with

$0.4<\zeta \leq 1.9$.

For $\zeta \lesssim 0.4$ the heating drives the decay completely and this formula is no longer applicable.

\subsection{Data analysis}

In our XMM-Newton observation the G9 dwarf star of the cluster ZS 76 showed two flares (Fig. 4). The first flare is already on at the beginning of the observation. The second flare started $\sim 15 \mathrm{ks}$ after the beginning of the observation, with a peak rate lower than that of the first flare and a rapid rise phase, lasting less than $800 \mathrm{~s}$. The e-folding decay times of the two flares are $\sim 1.4$ and $\sim 1.5 \mathrm{ks}$, respectively. The first flare shows two different decay trends, initially slower, then faster (Fig. 4); in the second flare the presence of a double decay is less clear. In order to trace the spectral changes during the flares, we binned the two flares into four and two temporal segments, respectively, marked in Fig. 4. The segments are chosen in a way to have a sufficient count statistics in the spectra. In the second flare only two segments can be built, with the spectra having 700-800 counts. The first flare can be binned into four segments, each with $\sim 1000$ counts. We recall that 
Table 1. Spectral fitting parameters for each temporal segment of the flares in star ZS76. In parentheses the $10 \%$ and $90 \%$ error ranges are reported. For A, B, C and E T and EM refer to the hot component of the 2-T model. For D segment $T$ and $E M$ refer to the hot component of the 3-T spectral model. For segment $\mathrm{F}$ of flare 2 a 2-T model is adopted with the only hot emission measure as free parameter. The $E M$ reported in this case is the difference with respect to the quiescent emission (see text).

\begin{tabular}{llllll}
\hline \hline Flare 1 & Segment & $\log T(\mathrm{~K})$ & $\log E M\left(\mathrm{~cm}^{-3}\right)$ & $\chi^{2}$ (d.o.f.) & $P\left(\chi>\chi_{0}\right)$ \\
\hline & $\mathrm{A}$ & $7.57(7.48-7.71)$ & $53.92(53.86-53.98)$ & $0.90(32)$ & $63 \%$ \\
& $\mathrm{~B}$ & $7.41(7.34-7.52)$ & $53.68(53.62-53.74)$ & $0.96(30)$ & $53 \%$ \\
& $\mathrm{C}$ & $7.37(7.26-7.54)$ & $53.42(53.30-53.50)$ & $0.85(32)$ & $71 \%$ \\
& $\mathrm{D}$ & $7.30(7.15-7.50)$ & $53.26(53.10-53.34)$ & $0.80(33)$ & $75 \%$ \\
\hline Flare 2 & Segment & $\log T(\mathrm{~K})$ & $\log E M\left(\mathrm{~cm}^{-3}\right)$ & $\chi^{2}($ d.o.f. $)$ & $P\left(\chi>\chi_{0}\right)$ \\
\hline & $\mathrm{E}$ & $7.48(7.38-7.58)$ & $53.62(53.54-53.68)$ & $0.78(24)$ & $76 \%$ \\
& F & $7.06-$ & $53.02(52.92-53.08)$ & $1.00(32)$ & $46 \%$ \\
\hline
\end{tabular}

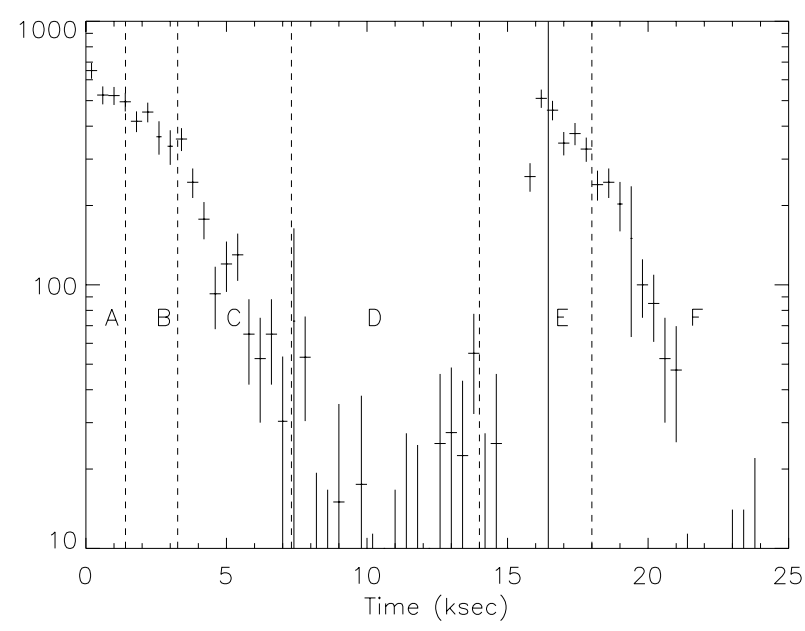

Fig. 4. Light curve of the flares observed in the star ZS 76. The dashed lines bound the time bins in which photon where collected for spectral analysis. The solid line marks the beginning of segment $\mathrm{E}$.

in Paper I we fitted the quiescent emission spectrum of ZS 76 with a 2-T component model obtaining the following parameters: $\log T_{1}=6.61, \log E M_{1}=53.03 \mathrm{~cm}^{-3}, \log T_{2}=7.06$, $\log E M_{2}=53.12 \mathrm{~cm}^{-3}$, abundance $Z=0.22 Z_{\odot}$ and $N_{\mathrm{H}}$ value fixed to $3 \times 10^{20} \mathrm{~cm}^{-3}$, in agreement with absorption estimate from optical photometry. We have fit the spectra in the time intervals $\mathrm{A}, \mathrm{B}, \mathrm{C}$ and $\mathrm{E}$ after having subtracted the quiescent spectrum. A single temperature model does not fit the data well, especially around $0.8-1.0 \mathrm{keV}$. A model with two absorbed APEC components yields a satisfactory agreement. The emission measure of the cooler component is much lower than that of the other component $(\sim 10 \%)$. The presence of this cool component is required to fit acceptably the region $0.8-1.0 \mathrm{keV}$ but plays no role for our physical interpretation of the flare. Therefore we will not discuss it further.

For interval D, due to the low count statistics, we have found one only way to obtain constrained parameters: we have considered a 3-T model in which the first two component temperatures and EMs are fixed to the quiescent values and the third component is free to vary and describes the flaring plasma. The global abundance is fixed to the quiescent value. For segment $\mathrm{F}$ we could only derive and show in Table 1 the flare component as the excess of emission measure over that of the hotter quiescent component, leaving only the hot $E M$ free to vary. The best fit parameters of spectral fitting are shown in Table 1. For intervals A, B, C and E the temperatures and EMs refer to the hot component in the 2-T model of flare spectrum. For D segment we report the parameters of the third conponent.

Figure 5 shows the evolution of the hardness ratio H/S during the first flare, where $\mathrm{H}$ and $\mathrm{S}$ are the count rates in the $0.75-2.4 \mathrm{keV}$ and $0.3-0.75 \mathrm{keV}$ bands, respectively. The bins in segments A, B and C are of $500 \mathrm{~s}$. For segment D, we show the average $\mathrm{H} / \mathrm{S}$ over the whole segment, to increase the statistical significance. The H/S evolution shows a very clear trend with two maxima in segments $\mathrm{A}$ and $\mathrm{C}$, which will be commented in Sect. 4.2. Figure 6 shows T vs. $\log E M^{1 / 2}$ for both flares. The dashed line marks a linear fitting of the decay of flare 1 ; its slope is $0.8 \pm 0.2$. Although evidence of reheating has been found in segment $\mathrm{C}$, and this may affect the description of the flare decay as a single decay, Reale et al. (2004) have shown that the description with a single decay is a reasonable approximation on the long time scale of the whole flare decay.

For flare 2 the path in $\log T$ vs. $\log E M^{1 / 2}$ has only two points in segments $\mathrm{E}$ and $\mathrm{F}$, which provide a rough estimate $\zeta \sim 1.3$.

\subsection{Results and discussion}

From the spectral fitting of segments A and E, we obtain that the maximum temperatures of the two flares are $\log T=7.61$ and $\log T=7.44$ dex, respectively. The spectral fitting detects the average temperatures of the flaring loops, which are therefore lower than the loop maximum temperatures. According to Reale et al. (2004) we can estimate the loop maximum temperatures $T_{\max }$ as $\approx 66 \mathrm{MK}$ and $\approx 52 \mathrm{MK}$, respectively; these values are intermediate between those of flares observed on active stars like Algol ( $T_{\max } \sim 100-150 \mathrm{MK}$, Favata et al. 2000b) and AB Dor $\left(T_{\max } \sim 140-170 \mathrm{MK}\right.$, Maggio et al. 2000), and those found in flares on the dMe star AD Leo (range 20-50 MK, Reale \& Micela 1998; Favata et al. 2000a) and on solar-type Pleiades (20-40 MK, Briggs \& Pye 2003). The EMs are of the same order as those estimated by Favata et al. (2000b) and Maggio et al. (2000) in Algol and AB Dor flares and larger than those of Pleiades flares reported by Briggs \& Pye (2003). 


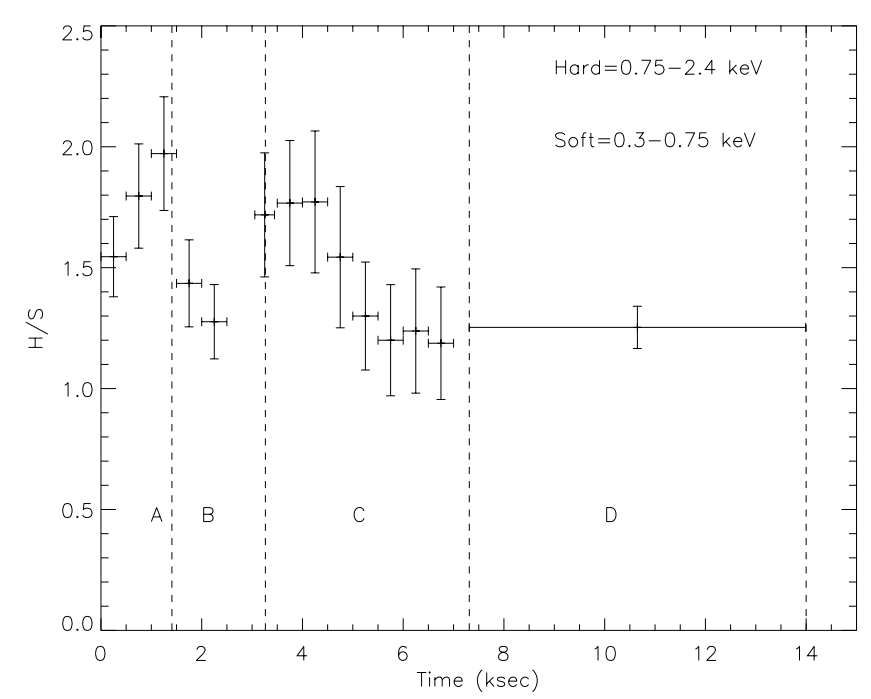

Fig. 5. Time evolution of hardness ratio $H / S(S=0.3-0.75 \mathrm{keV}$; $H=0.75-2.4 \mathrm{keV}$ ) of flare 1 . In segments $\mathrm{A}, \mathrm{B}$ and $\mathrm{C}$ the time bins are $500 \mathrm{~s}$; in segment $\mathrm{D}$ the average $H / S$ of the whole segment is shown to increase the statistical significance.

According to Eq. (1), the slope of the decay path shown in Fig. 6 and the value of the light curve decay time lead to a loop semi-length $L \approx 8 \times 10^{9} \mathrm{~cm}$ with uncertainties $\sim 50 \%$, for flare 1 . For flare 2 the decay time and the estimated value of $\zeta$ lead to a similar loop length, but we cannot quote any confidence for this value. The global similarity of the two flares supports the hypothesis that they occur in similar loop structures, or, maybe, in the same one, and we will make our further arguments in this perspective.

The trend in $\log T$ vs. $\log E M^{1 / 2}$ suggests the presence of significant heating during the decay of the first flare. Its hardness ratio (Fig. 5) indicates that a heat pulse has occurred in segments $\mathrm{A}$ and another one in segment $\mathrm{C}$. The time delays between the heating pulses in this flare are of the order of $2 \mathrm{ks}$.

The loop semi-lengths are a factor $\sim 8$ smaller than the stellar radius (estimated to be $\sim 6 \times 10^{10} \mathrm{~cm}$, by following isochrone model of Siess et al. 2000). On the other hand, the large EMs derived from spectral fitting in segments $\mathrm{A}$ and $\mathrm{E}$ (a few times $\sim 10^{53} \mathrm{~cm}^{-3}$ ) indicate either high densities or large volumes (or both). In order to estimate the loop volume, we have to make an assumption for the loop aspect ratio $r / L$ where $r$ is the radius of the loop cross-section, assumed circular. If we assume $r / L=0.1$, typical of solar single loops, we obtain a volume of $\sim 3 \times 10^{28} \mathrm{~cm}^{-3}$ and a density at the flare peak $\gtrsim 5 \times 10^{12} \mathrm{~cm}^{-3}$, significantly high for a plasma in coronal conditions. Furthermore, with the single loop volume obtained above, the total input energy rate $H_{\text {tot }}$ at the flare peak would be $\sim 12 \%$ of the maximum flare luminosity $L_{X}$, while we expect it to be larger than $L_{X}$ to satisfy the flare energy balance.

If we suppose that at the flare peak the loop is not too far from energy equilibrium, the loop scaling laws of Rosner et al. (1978) - which link the pressure, the loop length and the heating rate per unit volume - should approximately hold. The densities obtained from scaling laws are more reasonably a few times $10^{11} \mathrm{~cm}^{-3}$ for both flares. For the density from the scaling laws to be consistent with the $E M$ at the peak, the volume
Table 2. Parameters derived for flares 1 and 2 from spectral fitting and from loop scaling laws. The uncertainty range of $T_{\max }$ is derived from the values in Table 1.

\begin{tabular}{lll}
\hline \hline & Flare 1 & Flare 2 \\
\hline$\tau_{\mathrm{LC}}(\mathrm{ks})$ & $1.4 \pm 0.2$ & $1.5 \pm 0.2$ \\
$\zeta^{(1)}$ & $0.8 \pm 0.2$ & $\sim 1.3$ \\
$T_{\max }^{(2)}\left(10^{6} \mathrm{~K}\right)$ & $66(52-95)$ & $52(40-68)$ \\
$L\left(10^{9} \mathrm{~cm}\right)$ & $8 \pm 4$ & $\sim 13$ \\
$L_{\mathrm{X}}^{(3)}\left(10^{30} \mathrm{erg} / \mathrm{s}\right)$ & 9.8 & 9.2 \\
$E_{\text {tot }}^{(4)}\left(10^{34} \mathrm{erg}\right)$ & 1.4 & 1.4 \\
$p^{(5)}\left(10^{3} \mathrm{dyn} / \mathrm{cm}^{2}\right)$ & 14 & 4 \\
$n_{\mathrm{e}}^{(6)}\left(10^{11} \mathrm{~cm}^{-3}\right)$ & 8 & 4 \\
$V^{(7)}\left(10^{30} \mathrm{~cm}^{3}\right)$ & 1.3 & 2.6 \\
$E_{\mathrm{H}}^{(8)}\left(10^{31} \mathrm{erg} / \mathrm{s}\right)$ & 5.3 & 1.6 \\
$N_{\text {loop }}^{(9)}$ & $\sim 30$ & $\sim 20$ \\
\hline
\end{tabular}

Notes:

(1) Slope of the decay in the $n-T$ diagram.

(2) Estimated loop maximum temperature at the flare peak.

(3) X-ray luminosity at the flare peak $(0.3-5 \mathrm{keV}$ band).

(4) Total emitted energy.

(5) Maximum pressure of the flaring loop estimated from the loop scaling laws (Rosner et al. 1978).

(6) Electron density at the location of the loop maximum temperature estimated from loop scaling.

laws (Rosner et al. 1978).

(7) Volume of the flaring region estimated from the maximum emission measure and from the estimated density.

(8) Maximum heating rate estimated from the loop scaling laws (Rosner et al. 1978) and assuming the volume $V$.

(9) Number of loops filling the flare volume, assuming an aspect $r / L=0.1$ for a single loop.

should be much larger than that estimated above, and in particular of the order of $10^{30} \mathrm{~cm}^{3}$ for both flares. The loop length can be made consistent with the large volume by supposing that the flaring structures are arcades of $\sim 20-30$ similar loops. Table 2 shows for each flare the e-folding time of the light curve decay, the slope of the decay in the $\log T$ vs. $\log E M^{1 / 2}$ diagram, the loop semi-length obtained from Eq. (1), the peak luminosity, the total emitted energy, the pressure, density, volume and total heating rate, derived assuming that the loop scaling laws approximately hold at the flare peak. In this scenario the maximum X-ray luminosity is (reasonably) about $15 \%$ of the predicted total input energy for both flares.

Similar loop systems have been observed to flare on the Sun, for instance during the so-called Bastille-day flare (see Aschwanden \& Alexander 2001), and a stellar analogue has been proposed to model a flare on the dMe star Proxima Centauri (Reale et al. 2004). In these two events, a double ignition in nearby loops was observed or suggested, and the delay between the ignitions appears to scale with the loop sizes (measured on the Sun, inferred from detailed modelling on Proxima Centauri). We then suggest that the similarity with solar arcade flares may extend to stellar flares in young, much more active stars. 


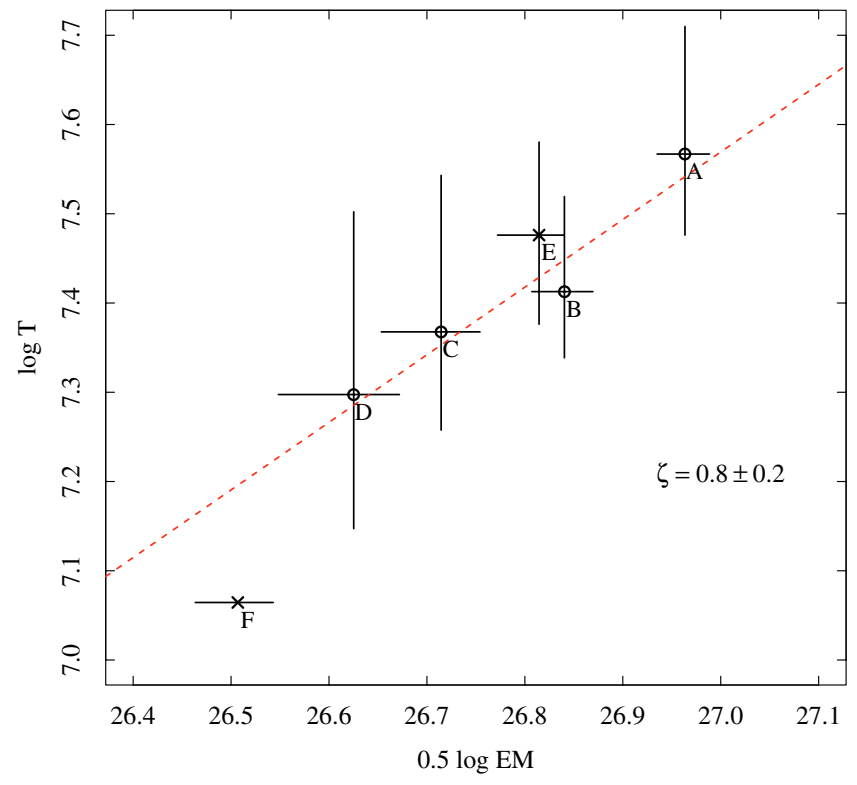

Fig. 6. Plot of $\log T$ vs. $\log E M^{1 / 2}$, obtained from the spectrum fit of the first flare of ZS76 (star symbols, points: A-D) and of the second flare (diamond symbols, points: E-F). Error bars are given as $10 \%-$ $90 \%$ range. The dashed line is the result of a linear fitting of the data points of flare 1 (A-D), its slope is $0.8 \pm 0.2$.. The slope of the segment that connects $\mathrm{E}$ and $\mathrm{F}$ points is 1.3 .

In summary, the data seem to be consistent with the occurrence of a double flare event, involving two arcades made of similar loops, or even the same arcade. If distinct, the short time distance between the two flares and their similarity may suggest that the two arcades are physically connected, the second triggered by the first, or may be part of a single complicated structure that requires some time to store new energy from the photospheric motions, to ignite once again in a similar way after several hours.

Also the flares observed on dM stars BLX 17, BLX46 and $B L X 37$ show secondary peaks (cf. Sect. 3) that may be consistent with the scenario, of series of loop systems ignited at subsequent time intervals. Hence the frequency of such gigantic multiple flares could be higher in young, active stars like those of Blanco 1, becoming one the most important causes of variability of stars with enhanced activity and indicating an age evolution of the flare activity from young to solar aged stars.

\section{Other X-ray variable sources}

This section gives some basic information about the remaining 8 out of 22 variable sources that do not belong to Blanco 1. Their light curves are shown in Fig. 7, and Table 3 lists their coordinates and the matches in the GSC2 and 2MASS catalogues within 13" as done in Paper I. In some cases double matches occur with an offset comparable to the EPIC PSF core and the choice of the most likely match is not obvious. The last column indicates the identifiers of objects already reported in literature, as found in SIMBAD database ${ }^{1}$. The only matching objects within $15^{\prime \prime}$ found in literature are from the paper of

\footnotetext{
${ }^{1}$ http://simbad.u-strasbg.fr/Simbad
}

Micela et al. (1999a) and, to date, no other studies in optical or infrared bands report other information about these X-ray sources. The 2MASS photometry of optical counterparts of X48 (closest match), X77 (closest match) and X150 is compatible with the cluster sequence at $\mathrm{dM}$ types, hence the X-ray activity and variability of these sources may suggest that they are unknown low mass stars of the cluster. In particular, X77 and $\mathrm{X} 150$ have proper motions compatible with those of the cluster, although their instrumental photometry from GSC2 catalog places these objects outside the cluster sequence. In almost all cases, the light curves show flare-like events, X77 and X151 also show some smooth modulation. The light curve of source X151 is strongly influenced by the nearby BLX 24 cluster star, so its variability is not firmly assessed. Furthermore, this source does not have a counterpart in the optical GSC2.2.1 and infrared 2MASS catalogs within 13" and does not appear in Table 3. The total spectrum of source X26 was reported in Paper I to be fitted with a 1-T APEC absorbed model at $\sim 0.7 \mathrm{keV}$. The absorption was $1.6 \times 10^{21} \mathrm{~cm}^{-2}$, significantly greater than the one for the cluster, while the abundance yielded a very low value to fit the spectrum $\left(Z=0.03 Z_{\odot}\right)$.

\section{Summary}

In this paper we have presented a study of the X-ray variability of the stars in the region of the young open cluster Blanco 1, observed with XMM-Newton satellite. The large effective area of the EPIC camera and the time coverage almost without interruption allow us for the first time to perform the detailed study of light curves of Blanco 1 stellar coronae. We have analyzed the X-ray variability of cluster coronae on the time scale of hours within the XMM-Newton observation, and on the time scale of a few years, by comparison with the data of previous ROSAT-HRI observations (Micela et al. 1999a). On the short time scale a large fraction of variable sources belong to the cluster. The variability is more frequent on late-types stars: the typical features are flare-like events among very low mass stars, while in solar-mass stars we have some indication of smooth variations. On the cluster star ZS76 the time resolved spectroscopy of two intense flares allows us to investigate the heating and the morphology of the flaring regions. To have consistency between the emission measure and temperature at the peak and the light curve time scales the flares should have occurred in two arcades made of several similar loops (half length: $\approx 10^{10} \mathrm{~cm}$ ), ignited in sequence and perhaps physically linked to each other. The occurrence of multiple flares in ZS76 and in the dM stars BLX 17, BLX 46 and $B L X 37$ may suggest that such flares may be common events on moderately active stars. The enhanced activity of young stars such those of Blanco 1 could also result in more crowded spatial coverage of their coronae, thus exhibiting multiple ignitions more frequently.

The comparison with ROSAT data shows that the variability on a time scale of $\sim 6$ yr appears very similar among $\mathrm{dF}-\mathrm{dM}$ stars, in spite of their spectral differences. Furthermore, the variations are within a factor 2 , while in an older star like the Sun, we observe variations as great as a factor of 10 , thus 
Table 3. Optical and infrared properties of $7 \mathrm{X}$-ray variable sources not belonging to the cluster, obtained through VizieR database. Source $\mathrm{X} 151$ does not match a counterpart in these catalogs and is not listed here. The coordinates are from X-ray detection, Cols. $r_{1}$ and $r_{2}$ are offset between the matching positions in arcsec. $R$ and $B_{j}$ magnitudes are from cross-identification with GSC 2.2.1 ( $\left.r \leq 13^{\prime \prime}\right)$ catalog. $J, H$, $K$ magnitudes are from cross-identification with 2MASS catalog. Column Notes reports the name of literature objects retrived from SIMBAD database within a $13^{\prime \prime}$ radius, for BLX31 the radius is $15^{\prime \prime}$.

\begin{tabular}{|c|c|c|c|c|c|c|c|c|c|c|}
\hline \multirow[t]{2}{*}{ Id } & \multirow{2}{*}{$\begin{array}{l}\text { RA } \\
\text { J2000 }\end{array}$} & \multirow{2}{*}{$\begin{array}{l}\text { Dec } \\
\text { J2000 }\end{array}$} & \multicolumn{3}{|l|}{ GSC 2.2.1 } & \multicolumn{4}{|c|}{ 2MASS } & \multirow[t]{2}{*}{ Notes } \\
\hline & & & $r_{1}\left({ }^{\prime \prime}\right)$ & $R$ (mag) & $B_{J}(\mathrm{mag})$ & $r_{2}\left({ }^{\prime \prime}\right)$ & $J$ (mag) & $H$ (mag) & $K$ (mag) & \\
\hline $\mathrm{X} 26$ & $00: 02: 39.2$ & $-30: 08: 04.7$ & 2.2 & 17.89 & - & 2.3 & 14.55 & 14.0 & 13.74 & BLX 22 \\
\hline X29 & $00: 03: 28.3$ & $-30: 07: 44.7$ & - & - & - & 2.6 & 16.31 & 15.59 & 15.50 & \\
\hline X48 & 00:02:07.6 & $-30: 04: 44.4$ & 2.0 & 16.69 & - & 1.9 & 13.95 & 13.26 & 13.15 & BLX 12 \\
\hline$"$ & "'" & 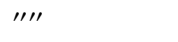 & 4.0 & 15.57 & 15.76 & 4.15 & 14.78 & 14.45 & 14.55 & \\
\hline X77 & 00:03:29.7 & $-30: 01: 18.9$ & 2.9 & 17.11 & 19.34 & 3.1 & 13.85 & 13.25 & 13.0 & BLX 45 \\
\hline$"$ & 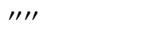 & "'"' & 6.0 & 17.76 & 19.04 & 5.5 & 16.70 & 16.13 & 15.33 & "'"' \\
\hline X83 & 00:02:09.4 & $-30: 00: 35.9$ & - & - & - & 1.5 & 15.21 & 14.70 & 14.38 & \\
\hline $\mathrm{X} 150$ & 00:03:22.6 & $-29: 53: 52.2$ & 2.3 & 15.82 & 17.89 & 2.4 & 12.98 & 12.35 & 12.15 & \\
\hline $\mathrm{X} 174$ & 00:03:00.7 & $-29: 49: 44.5$ & 2.8 & 17.27 & 19.30 & 2.5 & 13.77 & 13.21 & 12.91 & BLX 31 \\
\hline
\end{tabular}
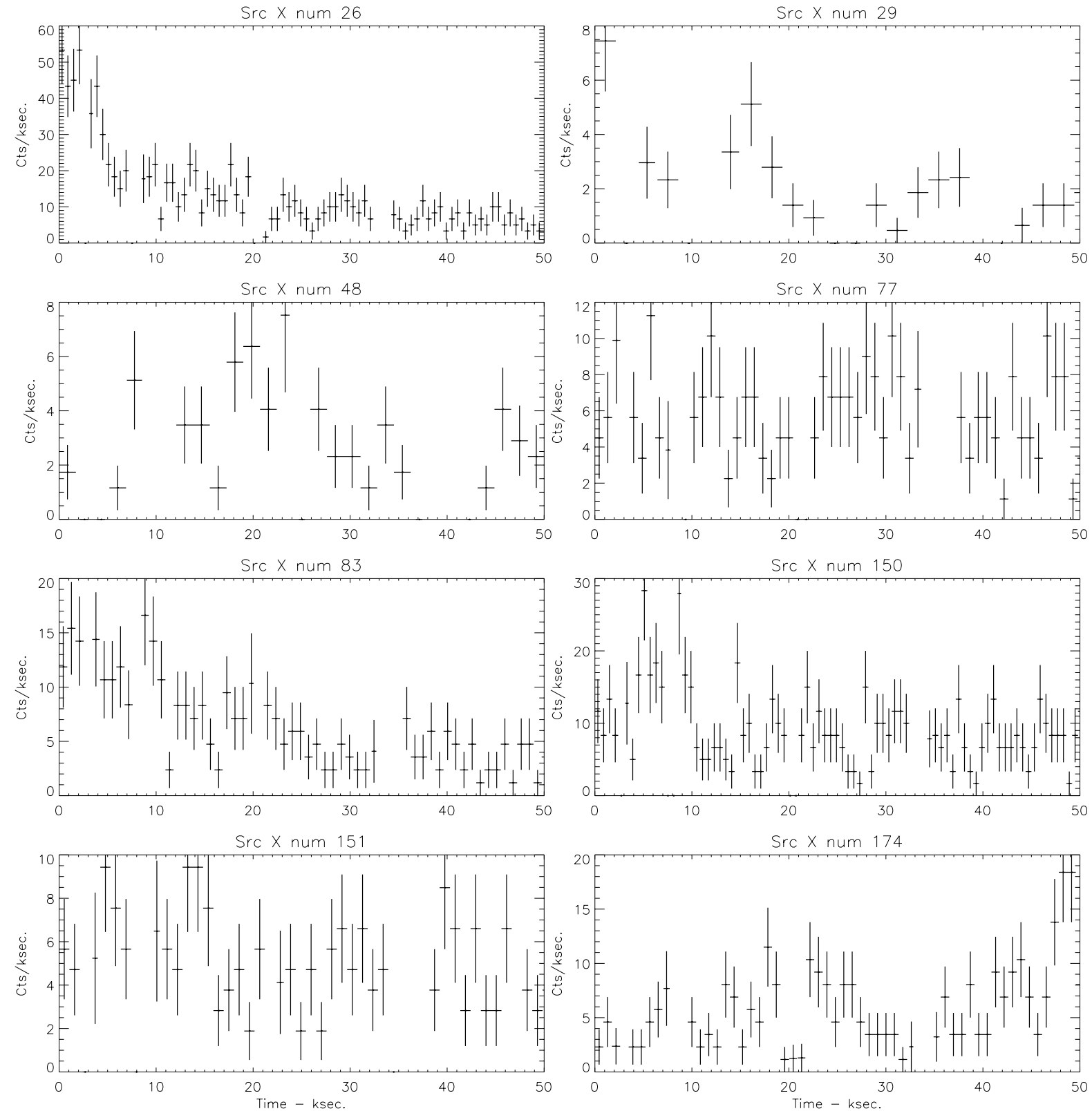

Fig. 7. Light curves of variable sources not belonging to Blanco 1. 
suggesting an age evolution of the emission mechanism from young to old, less active stars.

Acknowledgements. The authors acknowledge the referee, Dr. M. Güdel, for comments that significantly improved the paper. Financial support from ASI (Italian Space Agency) and MIUR (Ministero dell'Istruzione, dell'Università e della Ricerca) is acknowledged.

\section{References}

Aschwanden, M. J., \& Alexander, D. 2001, Sol. Phys., 204, 91 Briggs, K. R., \& Pye, J. P. 2003, MNRAS, 345, 714

Edvardsson, B., Pettersson, B., Kharrazi, M., \& Westerlund, B. 1995, A\&A, 293, 75

Favata, F., Micela, G., \& Reale, F. 2000a, A\&A, 354, 1021

Favata, F., Micela, G., Reale, F., Sciortino, S., \& Schmitt, J. H. M. M. 2000b, A\&A, 362, 628

Favata, F., Micela, G., \& Reale, F. 2001, A\&A, 375, 485

Gagné, M., Caillault, J., \& Stauffer, J. R. 1995, ApJ, 450, 217

Jardine, M. 2004, A\&A, 414, L5

Jeffries, R. D., Thurston, M. R., \& Pye, J. P. 1997, MNRAS, 287, 350

Maggio, A., Pallavicini, R., Reale, F., \& Tagliaferri, G. 2000, A\&A, 356,627
Marino, A., Micela, G., Peres, G., \& Sciortino, S. 2003, A\&A, 407, L63

Micela, G., \& Marino, A. 2003, A\&A, 404, 637

Micela, G., Sciortino, S., Favata, F., Pallavicini, R., \& Pye, J. 1999a, A\&A, 344, 83

Micela, G., Sciortino, S., Harnden, et al. 1999b, A\&A, 341, 751

Pillitteri, I., Micela, G., Sciortino, S., \& Favata, F. 2003, A\&A, 399, 919

Pillitteri, I., Micela, G., Sciortino, S., Damiani, F., \& Harnden, F. R. 2004, A\&A, 421, 175

Reale, F., \& Micela, G. 1998, A\&A, 334, 1028

Reale, F., Betta, R., Peres, G., Serio, S., \& McTiernan, J. 1997, A\&A, 325,782

Reale, F., Güdel, M., Peres, G., \& Audard, M. 2004, A\&A, 416, 733

Rosner, R., Tucker, W. H., \& Vaiana, G. S. 1978, ApJ, 220, 643

Serio, S., Reale, F., Jakimiec, J., Sylwester, B., \& Sylwester, J. 1991, A\&A, 241, 197

Siess, L., Dufour, E., \& Forestini, M. 2000, A\&A, 358, 593

Stelzer, B., Burwitz, V., Audard, M., et al. 2002, A\&A, 392, 585

Stern, R. A., Schmitt, J. H. M. M., \& Kahabka, P. T. 1995, ApJ, 448,683

Sylwester, B., Sylwester, J., Serio, S., et al. 1993, A\&A, 267, 586 